\title{
MiR-494 alleviates lipopolysaccharide (LPS)-induced autophagy and apoptosis in PC-12 cells by targeting IL-13
}

\author{
Wei Geng ${ }^{1, A, C-F}$, Lei Liu ${ }^{2, B, D, F}$ \\ ${ }^{1}$ Department of Spine Surgery, Liaocheng People's Hospital, China \\ ${ }^{2}$ Department of Orthopedic Surgery, Liaocheng $3^{\text {rd }}$ People's Hospital, China \\ A - research concept and design; $\mathrm{B}$ - collection and/or assembly of data; $\mathrm{C}$ - data analysis and interpretation; \\ $D$ - writing the article; $E$ - critical revision of the article; $F$ - final approval of the article
}

\section{Address for correspondence \\ Wei Geng}

E-mail: gengwei159@126.com

Funding sources

None declared

Conflict of interest

None declared

Received on March 28, 2017

Reviewed on July 31,2017

Accepted on September 1, 2017

Published online on August 29, 2018

\begin{abstract}
Background. MiR-494 is reported to act as a tumor-suppressive factor that inhibits the proliferation and colony formation of some cancer cells. However, there is still no report available on miR-494 functions in spinal cord injury (SCI) until now.

Objectives. The objective of this study was to examine the status of miR-494 in PC-12 cells injury induced by lipopolysaccharide (LPS), as well as its mechanism.
\end{abstract}

Material and methods. The cell counting kit-8 (CKK-8) assay and apoptosis assay were respectively used to determine the proliferation and apoptosis of $\mathrm{PC}-12$. The reverse transcription polymerase chain reaction (RT-PCR) analysis and western blot analysis displayed the expression of related factors at mRNA and protein level, respectively.

Results. The results showed that LPS could significantly decrease cell viability, and promote the cell apoptosis and autophagy of $\mathrm{PC}-12$ in a dose-dependent manner $(p<0.05)$. The overexpression of miR-494 could protect PC-12 cells from LPS-induced injury, as miR-494 overexpression increased cell viability, and reduced cell apoptosis and autophagy $(p<0.05)$. MiR-494 played a negative regulatory role in interleukin (IL)-13, and IL-13 was a direct target of miR-494. The overexpression of IL-13 could significantly aggravate LPS-diminished cell viability, and LPS-induced apoptosis and autophagy $(p<0.05)$. Besides, the overexpression of miR-494 did not attenuate LPS-induced injury when IL-13 was overexpressed. Furthermore, we found that the overexpression of miR-494 could significantly promote the phosphorylation of STAT6/MAPK and ERK/JNK signaling pathway.

Conclusions. MiR-494 could protect PC-12 cells from LPS-induced cell damage by targeting IL-13, and the activation of STAT6/MAPK and ERK/NKK pathways.

Key words: apoptosis, interleukin-13, autophagy, miR-494, PC-12

Cite as

Geng W, Liu L. MiR-494 alleviates lipopolysaccharide (LPS)-induced autophagy and apoptosis in P(-12 cells by targeting IL-13. Adv Clin Exp Med. 2019;28(1):85-94. doi:10.17219/acem/76749

DOI

10.17219/acem/76749

\section{Copyright}

Copyright by Author(s)

This is an article distributed under the terms of the

Creative Commons Attribution Non-Commercial License

(http://creativecommons.org/licenses/by-nc-nd/4.0/) 


\section{Introduction}

Spinal cord injury (SCI), characterized by a high disability rate, high mortality rate and poor prognosis, is a serious disease of the central nervous system. ${ }^{1,2}$ It is caused by lateral bending, axial loading, rotation, dislocation, and hyperextension or hyperflexion of the spinal cord, leading to the dysfunction of locomotion and paralysis. Spinal cord injury can cause damage to body functions, such as loss of feeling, abnormal reflexes, movement disorders, impairment of bowel and bladder control, and other changes. This damage cause permanent disability and many people are unable to take care of themselves. In China, nearly 60,000 new cases occur annually and the figure is rising. Unfortunately, the majority of the victims are young, between 15 and 40 years of age. Spinal cord injury brings enormous emotional and financial burdens to the patients, their families and the society. ${ }^{1}$ The precautions, treatment and rehabilitation of SCI have been a question for study in the domain of medical science.

The pathological process of SCI involves the primary injury and secondary injury. ${ }^{3}$ The primary injury is defined as damage to the spinal cord by mechanical force directly, such as distraction, transaction, compression, and laceration. The secondary injury that results in the evolution of physiological and biochemical reactions, such as edema, ischemia, immune responses, excitotoxicity, and electrolyte disturbances, follows the primary injury. ${ }^{4}$ Both hypoxia-ischemia and inflammation can cause the production and release of reactive oxygen species (ROS), and massive accumulation of ROS, which leads to the oxidative injury of local tissues, as well as the apoptosis and necrosis of neurons. Therefore, it is of extreme importance to study the molecular mechanism of ROS damage following SCI.

In normal tissue, cell proliferation and growth should coexist with aging and death. There are 3 categories of cell death: apoptosis, autophagic cell death and necrosis. The former 2 deaths are also known as programmed cell death. Large empirical studies have confirmed that apoptosis and autophagy were the significant cell death form in secondary SCI. ${ }^{5}$ Apoptosis is a process of programmed cell death, which is accurately regulated by genetic information. Once the balance between cell proliferation and apoptosis has been broken, it leads to a tumor or autoimmunity disease, or other diseases.

Caspase-3, also named as cysteine protease P32, is generally accepted as the key protease in cell apoptosis. Posttranslational activation of caspase- 3 requires proteolytic cleavage of the precursor protein and is generated into 2 subunits, which leads to enzyme polymerase activation and the initialization of apoptosis. According to previous reports, caspase-3 involves neuronal apoptosis in secondary $\mathrm{SCI}$, and the expression level of caspase- 3 is positively correlated with the apoptosis level of nerve cells. ${ }^{6}$ In addition, the anti-apoptotic Bcl-2 and the pro-apoptotic Bax also play important roles in regulating cell death. ${ }^{7,8}$ Several lines of evidence indicate that the relative ratio of these 2 proteins can determine, to a great extent, the response of an individual cell to the apoptotic process. ${ }^{9,10}$ The expression of $\mathrm{Bcl}-2$ and $\mathrm{Bax}$, and the ratio between $\mathrm{Bcl}-2$ and Bax could regulate the apoptosis.

MicroRNAs (miRNAs) are a class of short non-coding RNAs, in the size range of 19-25 nucleotides, which regulate the expression of target genes at the post-transcriptional level by combining 3' UTR of target genes and causing translational inhibition and mRNA degradation. ${ }^{11}$ Several studies have demonstrated that miRNAs play a key role in various cellular processes, such as cell cycle, proliferation, migration, differentiation, and tumorigenesis. ${ }^{12}$ In recent years, a large number of researches have shown that miRNA plays important roles in the development and progression of malignant tumors. ${ }^{13-15}$ Of note are the miRNAs that play important roles not only in human cancers, but also in cardiovascular disease, including cardiac hypertrophy, heart failure and ischemic heart disease. ${ }^{16}$

It has been reported that miR-494 could suppress the proliferation of some cancer cells, such as gastric cancer, lung cancer,and head and neck squamous cell carcinoma cells. ${ }^{17-19}$ However, there has been no report available on miR-494 functions in SCI until now. In this study, we aimed to explore the effects of miR-494 on lipopolysaccharide (LPS)-injured PC-12 cells by the detection of cell viability and apoptosis, autophagy, as well as STAT6/MAPK and ERK/JNK signaling pathways. The findings of this study can provide us with a new perspective suggesting that miR-494 might exert a protective role in LPS-damaged PC-12 cells.

\section{Material and methods}

\section{Cell culture and treatment}

The PC-12 cells were purchased from the Kunming Institute of Zoology (China) and used throughout the study. The cells were seeded onto flasks at a density of $1 \times 10^{4}$ cells/mL in Dulbecco's Modified Eagle Medium (DMEM) (Sigma-Aldrich, St. Louis, USA) with $10 \%$ (v/v) fetal bovine serum (FBS), $100 \mathrm{U} / \mathrm{mL}$ penicillin and $100 \mu \mathrm{g} / \mathrm{mL}$ streptomycin. The culture was maintained at $37^{\circ} \mathrm{C}$ in a humidified incubator containing $5 \%$ carbon dioxide $\left(\mathrm{CO}_{2}\right)$. The culture medium was changed every other day. The $3 \%$ and $21 \%$ oxygen $\left(\mathrm{O}_{2}\right)$ concentration were as the hypoxia and normoxia culture conditions. The culture medium was changed every other day.

Cells were treated by LPS in a series of concentrations for $12 \mathrm{~h}$.

Cells were treated with STAT6 inhibitor AS1517499 (100 nM), p38-MAPK inhibitor SB203580 (20 $\mu \mathrm{M})$, JNK inhibitor SP600125 (100 nM), or ERK inhibitor PD98059 $(20 \mu \mathrm{M})$ for $1 \mathrm{~h}$. 


\section{Cell counting kit-8 assay}

Cells were seeded in a 96-well plate with 5000 cells/ well. Cell proliferation was assessed by a cell counting kit-8 - CCK-8 (Dojindo Molecular Technologies, Gaithersburg, USA). Briefly, after stimulation, the CCK-8 solution was added to the culture medium, and the cultures were incubated for $1 \mathrm{~h}$ at $37^{\circ} \mathrm{C}$ in humidified $95 \%$ air and $5 \% \mathrm{CO}_{2}$. The absorbance was measured at $450 \mathrm{~nm}$ using a microplate reader (Bio-Rad Laboratories, Hercules, USA).

\section{Apoptosis assay}

Flow cytometry analysis was performed to identify and quantify the apoptotic cells by using the Annexin-Vfluorescein isothiocyanate (FITC)/propidium iodide (PI) apoptosis detection kit (Beijing Biosea Biotechnology, Beijing, China). The cells $(100,000$ cells/well) were seeded in a 6-well plate. Treated cells were washed twice with cold phosphate-buffered saline (PBS) and resuspended in a buffer. The adherent and floating cells were combined and treated according to the manufacturer's instruction, and measured with a flow cytometer (Beckman Coulter, Brea, USA) to differentiate apoptotic cells (Annexin-Vpositive and PI-negative) from necrotic cells (Annexin-V and PI-positive).

\section{Transfection and generation of stably transfected cell lines}

MiR-494 mimics, inhibitors and their respective negative controls (NC) were synthesized (Life Technologies Corporation, Carlsbad, USA) and transfected into cells in the study. The sequences of the oligonucleotides used were as follows: miR-494 mimic; sense 5'-UGAAACAUACACGGGAAACCUC-3'; antisense 5'-GGUUUCCCGUGUAUGUUUCAUU-3'; mimic control; sense 5'-UUCUCCGAACGUGUCACGUUU-3'; antisense 5'-ACGUGACACGUUCGGAGAAUU-3'; miR-494 inhibitor 5'-GAGGUUUCCCGUGUAUGUUUCA-3'; and inhibitor control 5'-UUCUCCGAACGUGUCACGUUUC-3'. For the analysis of the interleukin (IL)-13 functions, the full-length IL-13 sequences and short-hairpin RNA directed against IL-13 were constructed in pEX-2 and U6/GFP/Neo plasmids (GenePharma, Shanghai, China), respectively. They were referred as to pEX-IL-13 and sh-IL-13. The Lipofectamine 3000 reagent (Life Technologies Corporation) was used for the transfection of the cells according to the manufacturer's instructions. The plasmid carrying a non-targeting sequence was used as the NC of sh-IL-13 that was referred as to sh-NC. The sequences of the shRNAs used were as follows: sh-IL-13, 5'AATGCCATCTACAGGACCCAG-3'; sh-NC, 5'-AAAGGTATGGTACGACGGCGT-3'. Since the highest transfection efficiency occurred at $48 \mathrm{~h}$, a 72 -hour post-transfection was considered as the harvest time in the subsequent experiments. The stably transfected cells were selected by the culture medium containing $0.5 \mathrm{mg} / \mathrm{mL}$ G418 (Sigma-Aldrich). After approx. 4 weeks, G418-resistant cell clones were established.

\section{Qualitative reverse transcription polymerase chain reaction}

Total RNA was extracted from cells and tissues using Trizol reagent (Life Technologies Corporation), according to the manufacturer's instructions. The TaqMan MicroRNA Reverse Transcription Kit and TaqMan Universal Master Mix II with the TaqMan MicroRNA Assay of miR494 and U6 (Applied Biosystems, Foster City, USA) were used for testing the expression levels of miR-494 in cells.

\section{Dual luciferase activity assay}

The 3'UTR target site was generated by polymerase chain reaction (PCR) and the luciferase reporter constructs with IL-13 3'UTR, carrying a putative miR-494 binding site into pMiR-report vector, were amplified by PCR. Cells were cotransfected with the reporter construct, control vector and miR-494 or scramble, using Lipofectamine 3000. Reporter assays were done using the dual luciferase assay system (Promega, Madison, USA), following the manufacturer's information.

\section{Western blot}

The protein used for western blotting was extracted using Radio Immunoprecipitation Assay (RIPA) lysis buffer (Beyotime Biotechnology, Shanghai, China) supplemented with protease inhibitors (Roche, Guangzhou, China). The proteins were quantified using the $\mathrm{BCA}^{\mathrm{TM}}$ Protein Assay Kit (Pierce, Appleton, USA). The western blot system was established using a Criterion XT Bis-Tris Gel system (Bio-Rad Laboratories), according to the manufacturer's instructions. The glyceraldehyde 3-phosphate dehydrogenase (GAPDH) antibody was purchased from SigmaAldrich. Primary antibodies were prepared in 5\% blocking buffer at a dilution of 1:1,000. Anti-Bcl-2 (ab59348), anti-Bax (ab32503), anti-pro-caspase-3 (ab32150), anticleaved-caspase-3 (ab13847), anti-caspase-9 (ab202068), anti-LC3B (ab48394), anti-Beclin-1 (ab62557), anti-p62 (ab56416), anti-IL-13 (ab106732), anti-STAT6 (ab32520), anti-p-STAT6 (ab28829), anti-p38MAPK (ab197348), antip-p38MAPK (ab47363), anti-ERK (ab54230), anti-p-ERK (ab214362), anti-p-c-Jun (ab32385), anti-c-Jun (ab31419), and anti-GAPDH (ab8245) were all obtained from Abcam (Cambridge, UK). Primary antibody was incubated with the membrane at $4^{\circ} \mathrm{C}$ overnight, followed by wash and incubation with secondary antibody, marked by horseradish peroxidase for $1 \mathrm{~h}$ at room temperature. After rinsing, the polyvinylidene difluoride (PVDF) membrane carried blots and the antibodies were transferred into the Bio-Rad ChemiDoc $^{\mathrm{Tm}}$ XRS system (Bio-Rad, Shanghai, China), and 
then $200 \mu \mathrm{L}$ of Immobilon Western Chemiluminescent HRP Substrate (Merck Millipore, Burlington, USA) was added to cover the membrane surface. The signals were captured and the intensity of the bands was quantified using Image Lab ${ }^{\mathrm{Tm}}$ Software (Bio-Rad, Shanghai, China).

\section{Statistical analysis}

All the experiments were repeated 3 times. Data is presented as the mean \pm standard deviation (SD). Statistical differences between the mean values of multiple groups were analyzed using SPSS v. 19.0 statistical software (IBM Corp., Armonk, USA). Statistical significance was analyzed by using analysis of variance (ANOVA), followed by the least significant difference (LSD(L)) test post-hoc. A p-value $<0.05$ was considered to indicate statistical difference.

\section{Results}

\section{Lipopolysaccharide inhibited cell proliferation, and promoted cell apoptosis and autophagy of PC-12}

As shown in Fig. 1A, the cell viability of PC-12 was significantly decreased as the concentrations of LPS increased ( $\mathrm{p}<0.05)$. On the contrary, the cell apoptosis rate of PC-12 was significantly increased as the concentrations of LPS increased ( $<$ < 0.05) (Fig. 1B). Western blot analysis (Fig. 1C) showed that the expression of $\mathrm{Bcl}-2$ was decreased, and the expression of Bax and cleaved-caspase-3/9 was increased as the concentrations of LPS increased. Moreover, we detected the expression of autophagy-related proteins. The results (Fig. 1D) showed that the expression of LC-II and Beclin-1 was increased, and the expression of p62 was decreased as the concentrations of LPS increased. This data indicated that LPS could promote cell apoptosis and autophagy of PC-12 in a dose-dependent manner, and $5 \mathrm{ug} / \mathrm{mL}$ was selected as a LPS-stimulatory condition which was used in this study.

\section{Overexpression of miR-494 alleviated cell damage induced by lipopolysaccharide}

As shown in Fig. 2A, transfection with miR-494 mimic could significantly increase the expression of miR-494, while miR-494 inhibitor could significantly decrease the expression of miR-494 ( $\mathrm{p}<0.05)$. To test the role of miR-494 in LPS-stimulated PC-12, the CCK- 8 and apoptosis assays were performed (Fig. 2B,C). We found that miR-494 overexpression significantly increased cell viability and decreased apoptosis under LPS treatment conditions $(\mathrm{p}<0.05)$. Contrastingly, miR-494 suppression decreased cell viability and increased apoptosis under LPS exposure $(\mathrm{p}<0.05)$. Western blot analysis showed that the over-expression of miR-494 could increase the expression of $\mathrm{Bcl}-2$ and p62, and decrease the expression of Bax, cleaved-caspase-3/9, LC3-II, and Beclin-1 (Fig. 2D,E). On the contrary, the expression of these proteins in miR-494 knock-down group showed the reverse trend. These results indicated that the overexpression of miR-494 could reduce the cell damage induced by LPS, while miR-494 knock-down could aggravate it.
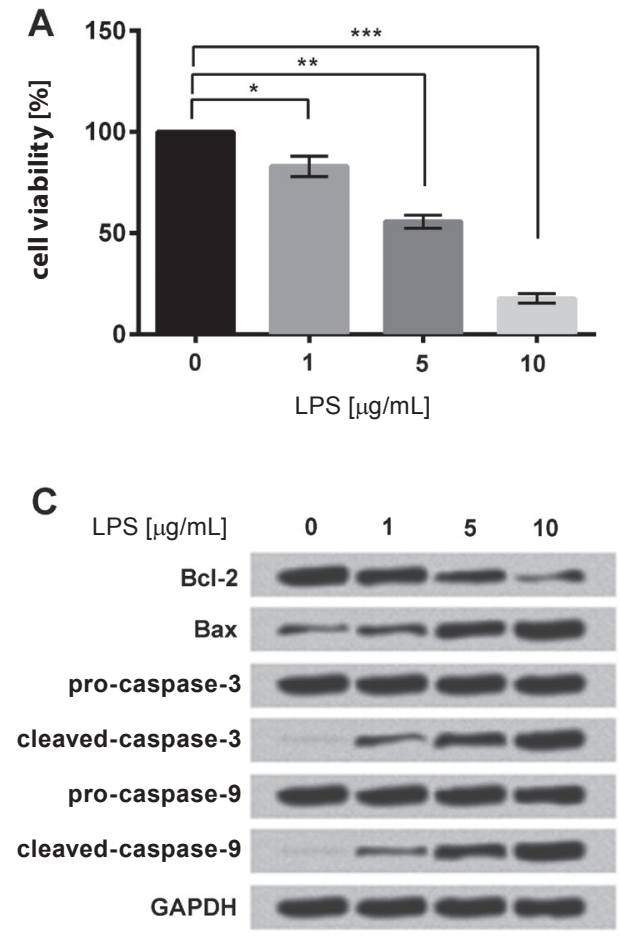

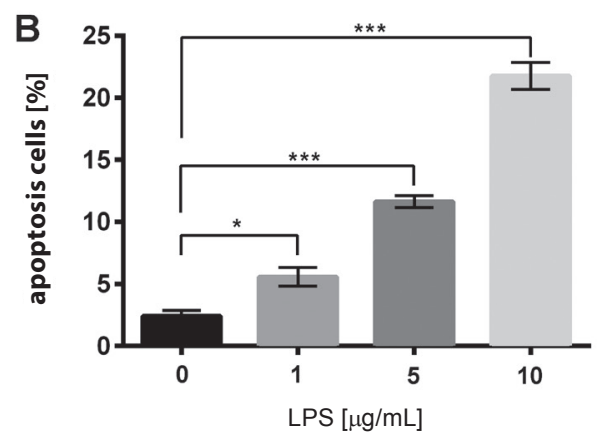

D

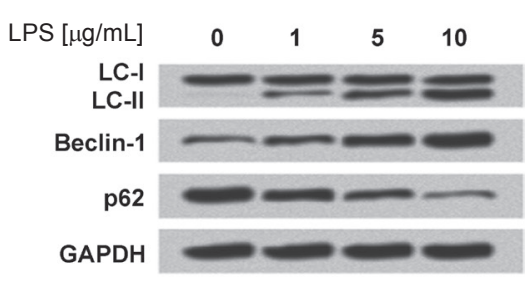

Fig. 1. Lipopolysaccharide affects cell proliferation, apoptosis and autophagy of PC-12

A - cell viability detected using MTT assay; B - apoptosis of PC-12 detected using apoptosis assay; C - apoptosis-related proteins detected using western blot; D - autophagy-related proteins detected using western blot.

LPS - lipopolysaccharide; MTT - methylthiazol tetrazolium; GAPDH - glyceraldehyde 3-phosphate dehydrogenase; data presented as mean $\pm S D$; ${ }^{*} p<0.05$; ** $p<0.01$; $* * * p<0.001$. 
A

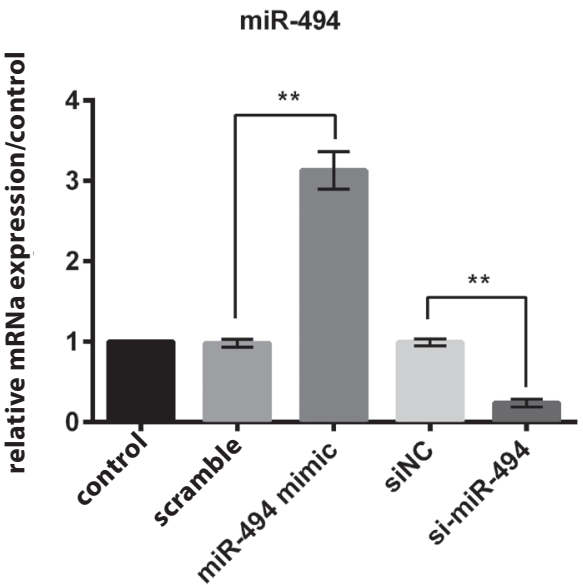

C

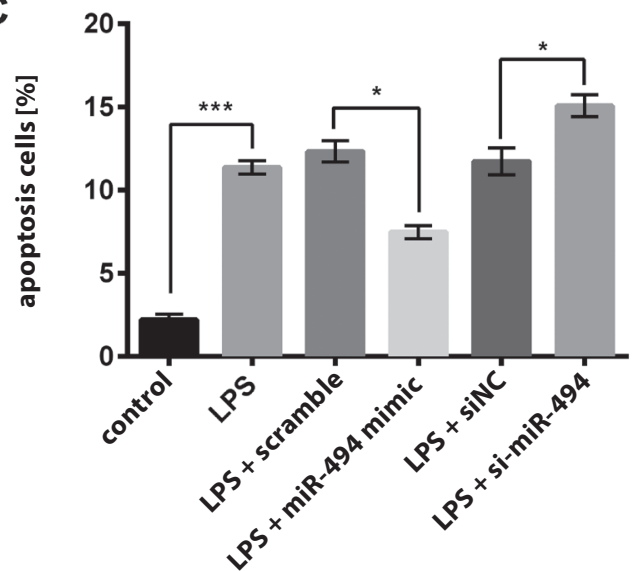

E

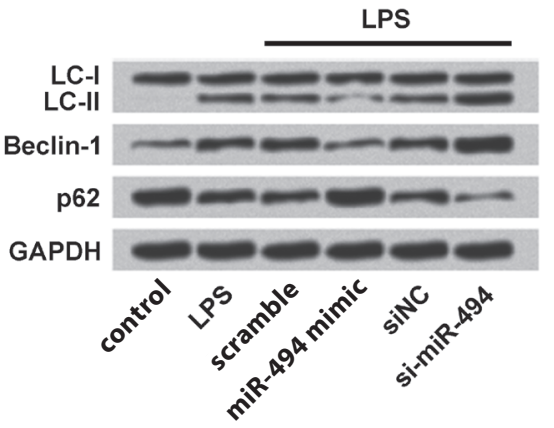

\section{Interleukin-13 was a direct target} of miR-494

As shown in Fig. 3A and 3B, the overexpression of miR494 could significantly decrease the expression of IL-13, while miR-494 knock-down could significantly increase the expression of IL-13 ( $\mathrm{p}<0.05$ ), indicating that miR494 acted as a negative regulation of IL-13. In addition, we found that the overexpression of miR-494 inhibited the luciferase activity of Wt-3'UTR of IL-13 by about $70 \%$ compared to the mutated miR-494 binding site (Fig. 3C). These results indicated that IL-13 was a direct target of miR-494.

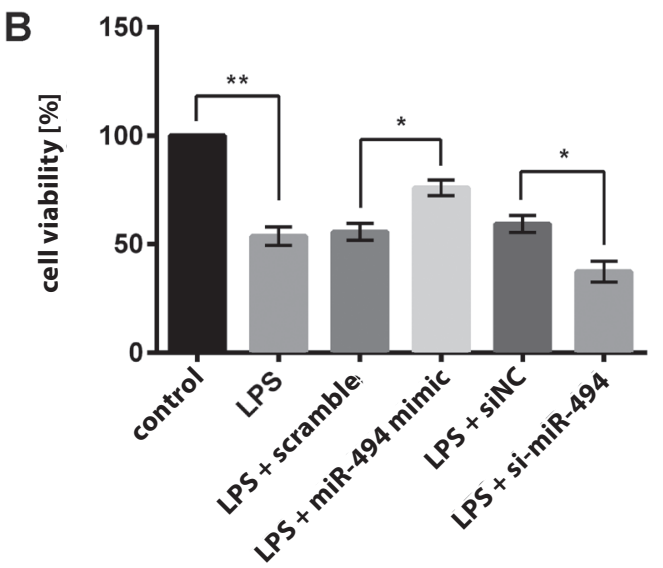

Fig. 2. MiR-494 affects cell proliferation, apoptosis and autophagy of $\mathrm{PC}-12$

A - expression of miR-494 detected using RT-PCR; $B$ - cell viability detected using MTT assay; $\mathrm{C}$ - apoptosis of PC-12 detected using apoptosis assay; D - apoptosis-related proteins detected using western blot; E - autophagyrelated proteins detected using western blot.

RT-PCR - reverse transcription polymerase chain reaction;

MTT - methylthiazol tetrazolium; GAPDH glyceraldehyde 3-phosphate dehydrogenase; data presented as mean $\pm \mathrm{SD}$; ${ }^{*} \mathrm{p}<0.05$; ${ }^{*} \mathrm{p}<0.01$; *** $p<0.001$.
LPS

D

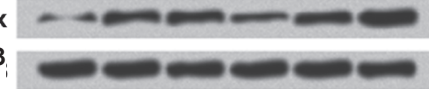

cleaved-caspase-3

pro-caspase-9,

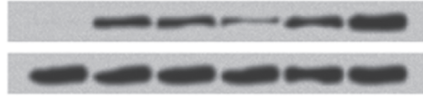

cleaved-caspase-9,

GAPDH

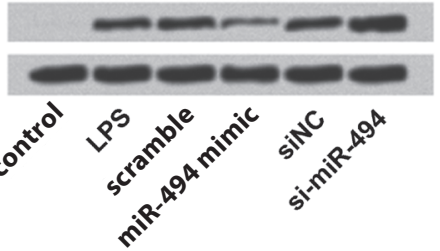

\section{Overexpression of miR-494 protected PC-12 cells from lipopolysaccharide- induced cell damage via targeting interleukin-13}

As shown in Fig. 4A and 4B, transfection with the IL-13 expressing vector, $\mathrm{pEX}-\mathrm{IL}-13$, could significantly increase the expression of IL-13, while transfection with IL-13 targeted shRNA, sh-IL-13, could significantly decrease the expression of IL-13 ( $p<0.05)$. We found that miR-494 overexpression did not prevent LPS-induced cell damage when IL-13 was overexpressed, as cell viability was reduced, Bcl-2 was downregulated, and Bax, cleaved-caspase-3/9 were upregulated after the addition of pEX-IL-13 ( $\mathrm{p}<0.05)$ (Fig. 4C,E). 


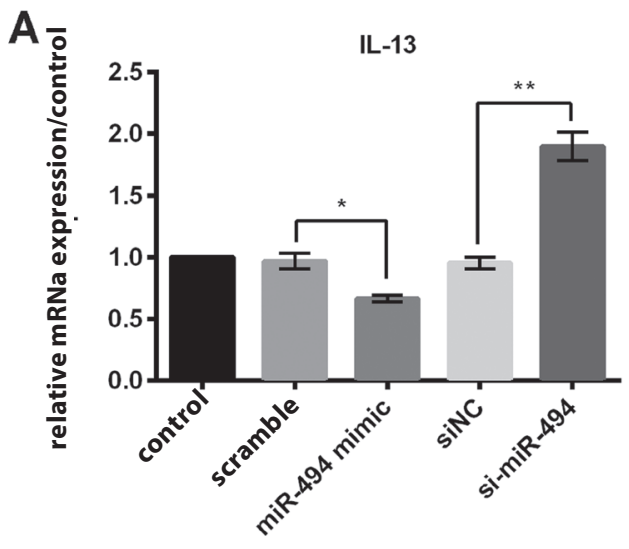

B
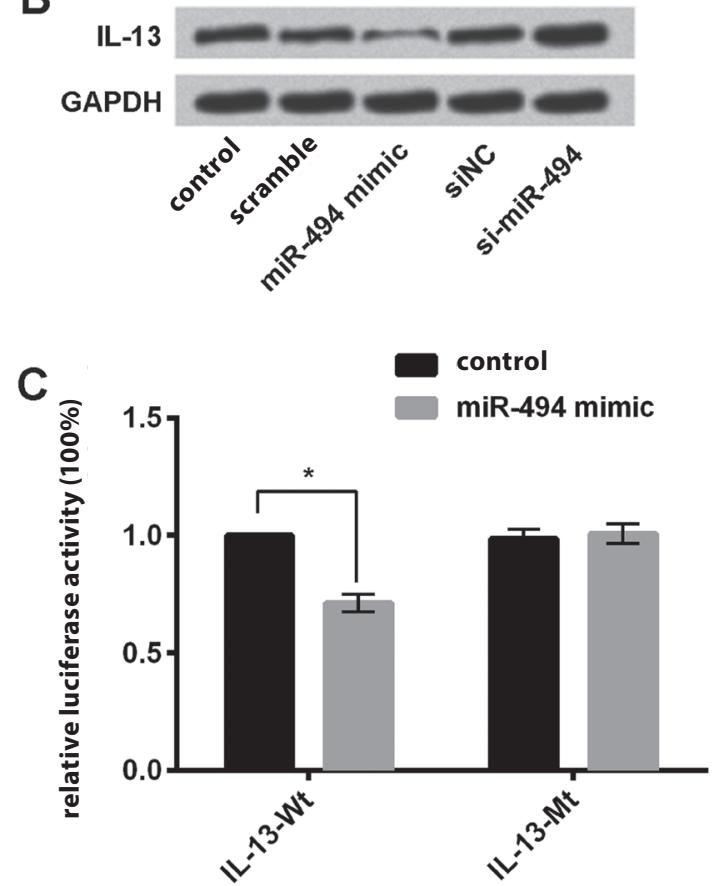

Fig. 3. IL-13 is a direct target of miR-494

A, B - expression of IL-13 detected with RT-PCR; C - dual luciferase assay performed on PC-12 cells transfected with luciferase construct alone or co-transfected with miR-494 mimics.

IL-13 - interleukin-13; RT-PCR - reverse transcription polymerase chain reaction; data presented as mean \pm SD; * $p<0.05$; ${ }^{* *} p<0.01$.

Meanwhile, miR-494 knock-down could not aggravate LPS-induced cell damage when IL-13 was silenced, as cell apoptosis was reduced, LC3-II and Beclin-1 were downregulated, while p65 was upregulated after the addition of sh-IL-13 (p < 0.05) (Fig. 4D,E). This data indicated that the overexpression of miR-494 protected PC-12 cells from LPS-induced cell damage via targeting IL-13.

\section{Interleukin-13 regulated cell damage of PC-12 induced by lipopolysaccharide}

As shown in Fig. 5A, the overexpression of IL-13 could significantly aggravate the proliferation inhibition of PC-12 induced by LPS ( $\mathrm{p}<0.05$ ), while the knock-down of IL-13 could significantly reduce the proliferation inhibition of PC-12 induced by LPS ( $\mathrm{p}<0.05)$. Figure $5 \mathrm{~B}$ showed that the overexpression of IL-13 could significantly promote the cell apoptosis of PC-12 induced by LPS ( $<<0.05$ ), while the knock-down of IL-13 could significantly inhibit the cell apoptosis of PC-12 induced by LPS ( $<<0.05)$. Western blot analytical results showed that IL-13 silence exerted the same effects as miR-494 overexpression on the expression of apoptosis and autophagy-related proteins (Fig. 5C,D), indicating the pro-apoptotic and pro-autophagic roles of IL-13 in PC-12 cells.

\section{MiR-494 activated the STAT6/MAPK and ERK/JNK signaling pathways}

Western blotting was used to examine the phosphorylation state of STAT6/MAPK and ERK/JNK in PC-12 cells that were treated with LPS (Fig. 6A,B). The results showed that the overexpression of miR-494 could significantly increase the expression of p-STAT6, p-p38MAPK, p-ERK, and p-c-Jun, while its knock-down could significantly decrease the expression of p-STAT6, p-p38MAPK, p-ERK, and $\mathrm{p}-\mathrm{c}$-Jun. These findings indicated that the knockdown of miR-494 inhibited STAT6/MAPK and ERK/JNK signaling pathway through upregulating the expression of IL-13, thus promoting the cell damage of PC-12 induced by LPS. Furthermore, to confirm this hypothesis, we measured the effect of miR-494 on the expression of p-STAT6, p-p38MAPK, p-ERK, and p-c-Jun after the inhibition of STAT6/MAPK and ERK/JNK pathways. As indicated by the results in Fig. $6 \mathrm{C}$ and $6 \mathrm{D}$, miR-494 overexpression did not upregulate p-STAT6, p-p38MAPK, p-ERK, or p-c-Jun when they were respectively exposed to STAT6 inhibitor AS1517499, p38-MAPK inhibitor SB203580, ERK inhibitor PD98059, or JNK inhibitor SP600125.

\section{Discussion}

Spinal cord injury is a common traumatic neuronal injury that imposes several complications and is a severe threat to human health. ${ }^{20}$ The microenvironment after SCI in adults is much less receptive to regrowth, thus hindering the regeneration of the damaged nerve. ${ }^{21}$ Therefore, the key method of improving SCI patient's outcomes is by utilizing protecting neurons, promoting the axonal regeneration. PC-12 cells are widely used as an in vitro neuron model for research on neurobiology, central nervous system diseases and neurotoxins. In the present study, we found that LPS could induce the cell damage of PC-12 via inhibiting cell proliferation, and promoting cell apoptosis and autophary, and the damage degree increased with increasing LPS concentration.

According to our data mentioned above, we found that LPS had a remarkable inhibition effect on the cell proliferation of PC-12, while promoting cell apoptosis and autophagy (Fig. 1). Apart from that, we found that LPS 
A

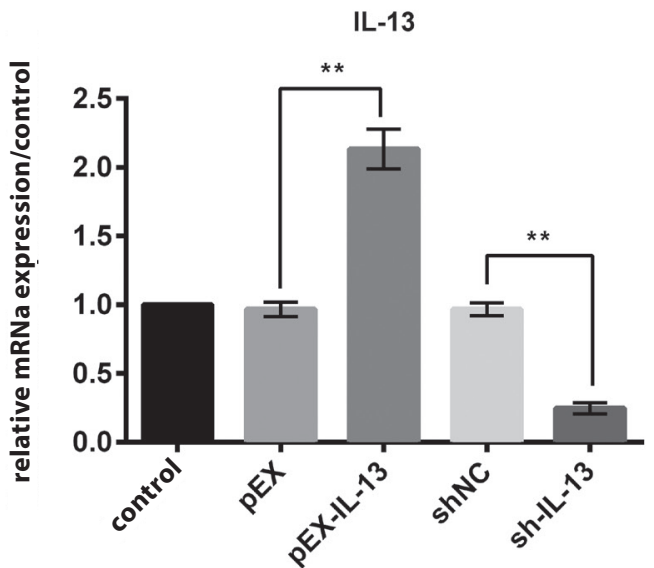

C

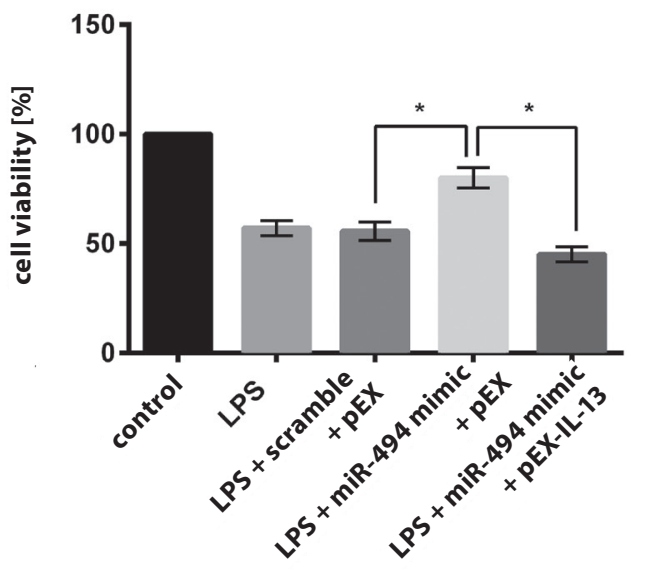

E

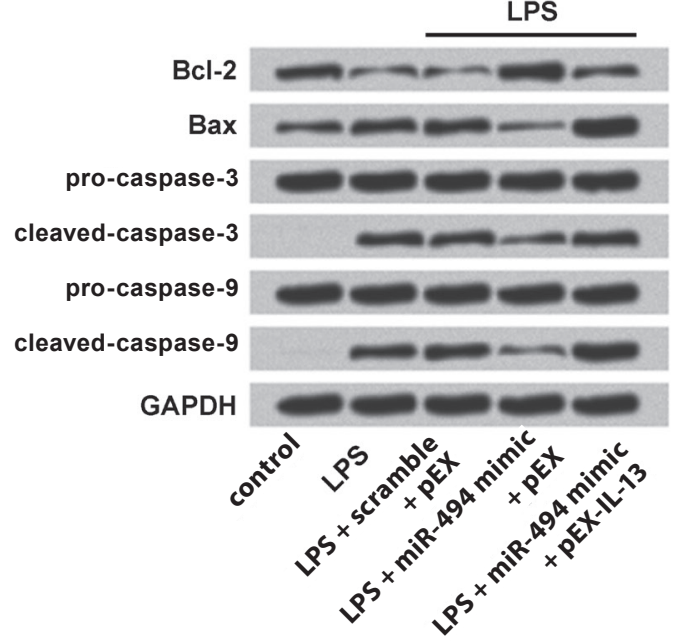

B
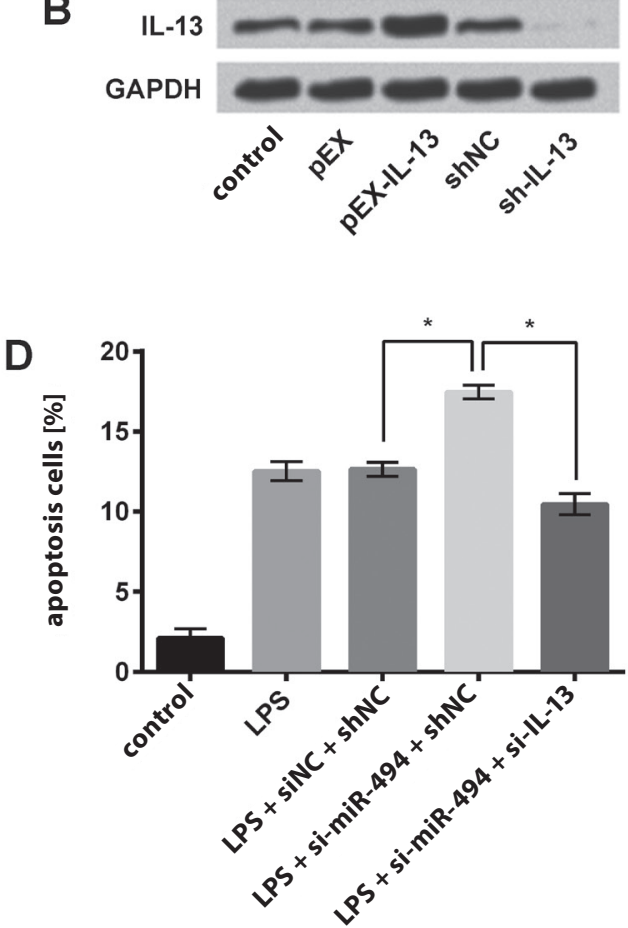

$\mathbf{F}$

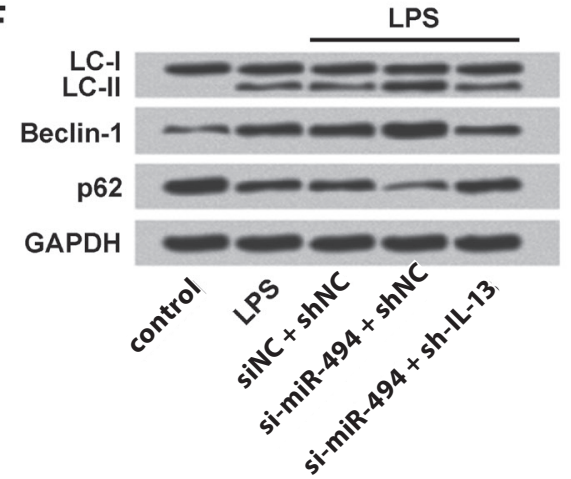

Fig. 4. Overexpression of miR-494 protected PC-12 cells from LPS-induced cell damage via targeting $\mathrm{IL}-13$

A, B - expression of IL-13 detected with RT-PCR; C - cell viability detected with MTT assay; D - apoptosis of PC-12 detected with apoptosis assay; E - apoptosis-related proteins detected with western blot; $\mathrm{F}$ - autophagy-related proteins detected with western blot.

LPS - lipopolysaccharide; IL-13 - interleukin-13; RT-PCR - reverse transcription polymerase chain reaction; MTT - methylthiazol tetrazolium; GAPDH glyceraldehyde 3-phosphate dehydrogenase; data presented as mean $\pm \mathrm{SD}$; ${ }^{*} p<0.05$; ${ }^{* *} p<0.01$. could decrease the expression of $\mathrm{Bcl}-2$, and increase the expression of Bax and caspase-3/9, thus promoting the apoptosis of PC-12. However, miR-494 could protect PC-12 cells from LPS-induced cell damage, as miR-494 overexpression increased cell viability, reduced apoptosis and autophagy, as well as increase the expression of $\mathrm{Bcl}-2$ and decrease the expression of Bax and caspase-3/9.

MiR-494, first reported to have a high expression in retinoblastoma, has been found to be implicated in various types of human cancers. ${ }^{22}$ In cancer, miR-494 functions as a tumor suppressor or oncogenic gene, dependent on different cell types. ${ }^{23}$ Apart from that, miR-494 was reported to act as a pivotal factor in regulating cell proliferation and apoptosis. ${ }^{24}$ In terms of SCI, miR-494 was reported to improve recovery from SCI and to attenuate apoptosis in SCI rats. ${ }^{25}$ Our study was consistent with this previous study, indicating that miR-494 exerted a protective function in SCI by reducing apoptosis, while our study provided the first evidence that miR-494 affected PC-12 cells also by controlling cell autophagy. 


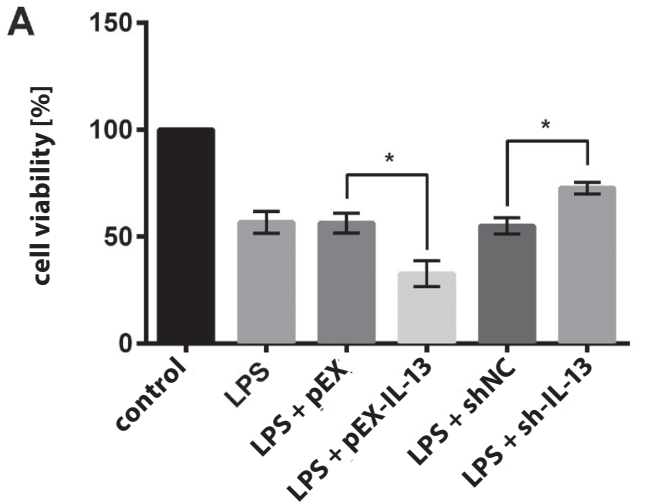

C

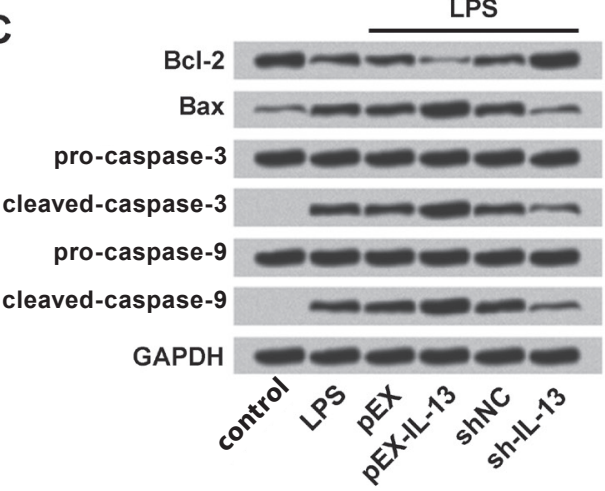

A

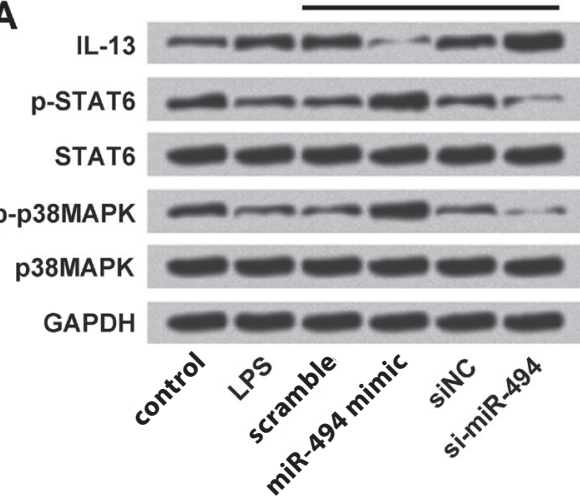

C

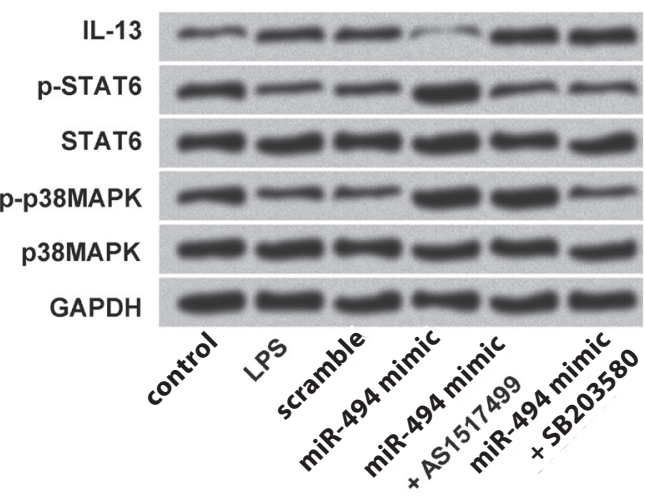

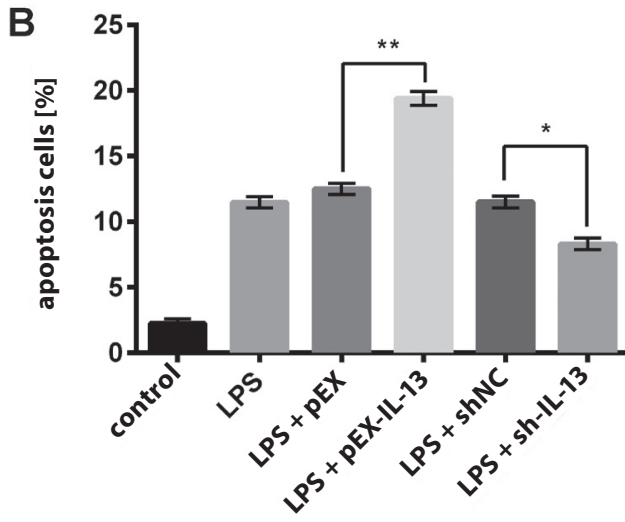

Fig. 5. IL-13 regulated cell damage induced by LPS

A - cell viability detected with MTT assay; B - apoptosis of PC-12 detected with apoptosis assay; C - apoptosis-related proteins detected with western blot; D - autophagy-related proteins detected with western blot.

IL-13 - interleukin-13; LPS

- lipopolysaccharide: MTT - methylthiazol tetrazolium; GAPDH - glyceraldehyde 3-phosphate dehydrogenase; data presented as mean $\pm S D$; ${ }^{*} p<0.05$ *** $p<0.01$.
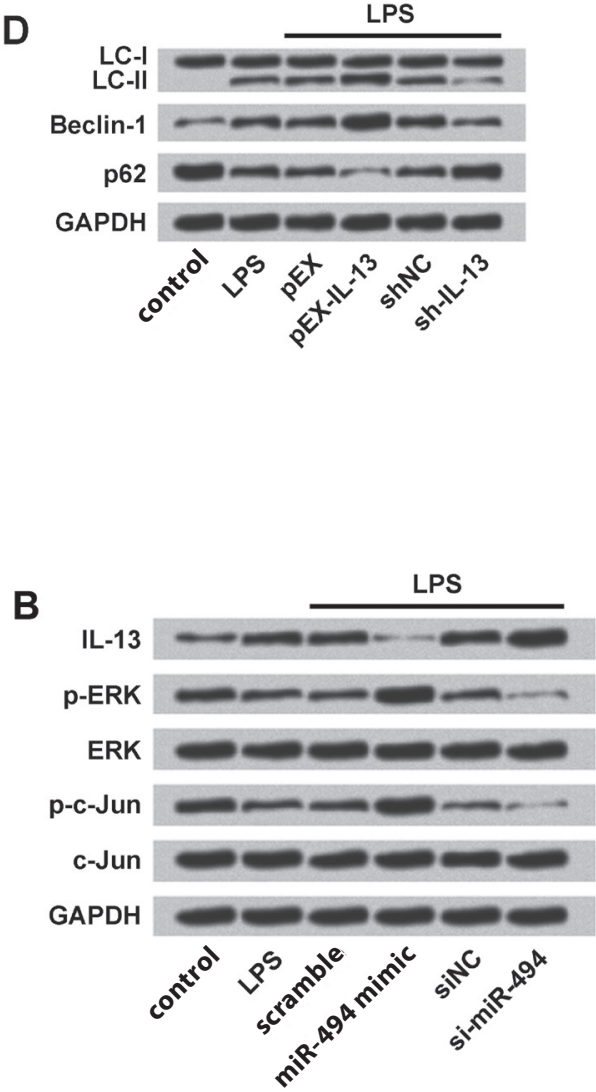

Fig. 6. MiR-494 affected expression of STAT6/MAPK and ERK/JNK pathways

\section{A, C - STAT6/MAPK signaling}

pathway-related proteins detected using western blot; B, D - ERK/JNK signaling pathway-related proteins detected using western blot.

GAPDH - glyceraldehyde

3-phosphate dehydrogenase:

LPS - lipopolysaccharide;

MAPK - mitogen-activated protein kinase; ERK - extracellular signal transduction kinase.
D

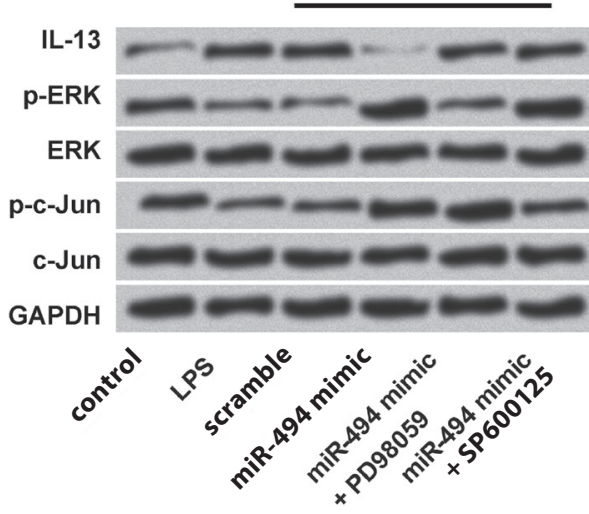


Autophagy is a unique life phenomenon of eukaryotes, which can degrade and recycle unnecessary or dysfunctional components. ${ }^{26,27}$ It plays an important role in the occurrence, development and treatment of the nervous system, digestive system and immune system diseases. Beclin-1 is an important factor in the initial period of autophagy, which is a mammalian ortholog of the yeast Atg6. ${ }^{28}$ With the action of Atg4, LC3 precursor is processed into soluble LC3-I, which combines with PE on the autophagic membrane, and then changes into LC3-II, which then attaches to the intracellular autophagic membrane until the autophagy-lysosome is formed. The LC3-II expression level is positively related to the sum of phagophores, suggesting that autophagy activity is closely related to the expression intensity of LC3-II. ${ }^{29,30}$ In addition, p62 located in the cytoplasm forms complexes by combining with ubiquitin protein and LC3-II protein. The complexes will eventually be degraded in the lysosome. ${ }^{31}$ Therefore, p62 is constantly consumed in the process of autophagy. These 3 proteins are usually recognized as the markers of the autophagy level. ${ }^{32-34}$ The above-mentioned results showed that LPS increased the expression of LC3-II and Beclin-1, and decreased the expression of p62, suggesting that LPS promoted PC-12 autophagy. However, the overexpression of miR-494 decreased the expression of LC3-II and Beclin-1, and increased the expression of $\mathrm{p} 62$, indicating that miR-494 could reduce the autophagy of PC-12 induced by LPS.

Mitogen-activated protein kinase (MAPK) is one kind of serine/threonine protein kinase which widely exists in eukaryotic cells. The family consists of extracellular signal transduction kinase (ERK), c-Jun-N-terminal kinase (JNK) and p38 kinase, etc. ${ }^{35}$ Extracellular signal transduction kinase mainly participates in cell proliferation, differentiation and transformation, while JNK and p38 are associated with intracellular and extracellular damage, infarct, inflammation, and oxidative stress reaction. ${ }^{36-38}$ It has been reported that MAPK pathways have anti-apoptotic effects in C6 glioma cells. ${ }^{39}$ In this paper, we found that the knock-down of miR-494 could inhibit the activation of STAT6/MAPK and ERK/JNK pathways, thus promoting LPS-induced cell damage.

In conclusion, our study suggested that miR-494 could protect PC-12 cells from LPS-induced cell damage by controlling cell proliferation, cell apoptosis and autophagy. The protective role of miR-494 in PC-12 cells might be carried out via targeting IL-13, and thus through the activation of STAT6/MAPK and ERK/JNK pathways. Our findings might provide better insight into the function of miR-494 in LPS-induced cell damage, which could contribute to clinical treatment of SCI.

\section{References}

1. Ackery A, Tator C, Krassioukov A. A global perspective on spinal cord injury epidemiology. J Neurotrauma. 2004;21(10):1355-1370.

2. Böthig R, Fiebag K, Thietje R, Faschingbauer M, Hirschfeld S. Morbidity of urinary tract infection after urodynamic examination of hospitalized SCI patients: The impact of bladder management. Spinal Cord. 2013;51(1):70.

3. Li S, Tator CH. Spinal cord blood flow and evoked potentials as outcome measures for experimental spinal cord injury. In: Stålberg E, Shanker Sharma H, Olsson Y, eds. Spinal Cord Monitoring: Basic Principles, Regeneration, Pathophysiology, and Clinical Aspects. Vienna, Austria: Springer; 1998:365-392.

4. Jones TB. Lymphocytes and autoimmunity after spinal cord injury. Exp Neurol. 2014;258:78.

5. Yune TY, Kim SJ, Lee SM, et al. Systemic administration of 17betaestradiol reduces apoptotic cell death and improves functional recovery following traumatic spinal cord injury in rats. J Neurotrauma. 2004;21(3):293-306.

6. Springer JE. Apoptotic cell death following traumatic injury to the central nervous system. J Biochem Mol Biol. 2002;35(1):94-105.

7. Kroemer G, Galluzzi L, Brenner C. Mitochondrial membrane permeabilization in cell death. Physiol Rev. 2007;87(1):99-163.

8. Green DR, Kroemer G. The pathophysiology of mitochondrial cell death. Science. 2004;305(5684):626-629.

9. Boise LH, González-García M, Postema CE, et al. Bcl-x, a Bcl-2-related gene that functions as a dominant regulator of apoptotic cell death. Cell. 1993;74:597-608.

10. Oltvai ZN, Milliman CL, Korsmeyer SJ. Bcl-2 heterodimerizes in vivo with a conserved homolog, Bax, that accelerates programmed cell death. Cell. 1993;74(4):609-619.

11. Garzon R, Pichiorri F, Palumbo T, et al. MicroRNA gene expression during retinoic acid-induced differentiation of human acute promyelocytic leukemia. Oncogene. 2007;26(28):4148-4157.

12. Schickel R, Boyerinas B, Park SM, Peter ME. MicroRNAs: Key players in the immune system, differentiation, tumorigenesis, and cell death. Oncogene. 2008;27(45):5959-5974.

13. Huang Y, Dai Y, Yang J, et al. Microarray analysis of microRNA expression in renal clear cell carcinoma. Eur J Surg Oncol. 2009;35(10): 1119-1123.

14. Shenouda SK, Alahari SK. MicroRNA function in cancer: Oncogene or a tumor suppressor? Cancer Metastasis Rev. 2009;28(3-4):369-378.

15. Calin GA, Croce CM. MicroRNA signatures in human cancers. Nat Rev Cancer. 2006;6(11):857-866.

16. Lv G, Shao S, Dong H, Bian X, Yang X, Dong S. MicroRNA-214 protects cardiac myocytes against $\mathrm{H} 2 \mathrm{O} 2$-induced injury. J Cell Biochem. 2014;115(1):93-101.

17. Kim WK, Park M, Kim YK, et al. MicroRNA-494 downregulates KIT and inhibits gastrointestinal stromal tumor cell proliferation. Clin Cancer Res. 2011;17(24):7584-7594.

18. Ohdaira H, Sekiguchi M, Miyata K, Yoshida K. MicroRNA-494 suppresses cell proliferation and induces senescence in A549 lung cancer cells. Cell Prolif. 2012;45(1):32.

19. Chang SS, Jiang WW, Smith I, et al. MicroRNA alterations in head and neck squamous cell carcinoma. Int J Cancer. 2008;123(12):2791.

20. Kang N, Hai Y, Yang J, Liang F, Gao CJ. Hyperbaric oxygen intervention reduces secondary spinal cord injury in rats via regulation of HMGB1/ TLR4/NF-KB signaling pathway. Int J Clin Exp Pathol. 2015;8(2): 1141-1153.

21. Nakamura M, Bregman B. Differences in neurotrophic factor gene expression profiles between neonate and adult rat spinal cord after injury. Exp Neurol. 2001;169(2):407-415.

22. Zhao JJ, Yang J, Lin J, et al. Identification of miRNAs associated with tumorigenesis of retinoblastoma by miRNA microarray analysis. Childs Nerv Syst. 2009;25(1):13-20.

23. Zhan MN, Yu XT, Tang J, et al. MicroRNA-494 inhibits breast cancer progression by directly targeting PAK1. Cell Death Dis. 2017;8(1): e2529.

24. Romano G, Acunzo M, Garofalo M, et al. MiR-494 is regulated by ERK1/2 and modulates TRAIL-induced apoptosis in non-small-cell lung cancer through BIM down-regulation. Proc Natl Acad Sci U S A. 2012;109(41):16570-16575. 
25. Gu S, Xie R, Liu X, Shou J, Gu W, Che X. Long coding RNA XIST contributes to neuronal apoptosis through the downregulation of AKT phosphorylation and is negatively regulated by miR-494 in rat spinal cord injury. Int J Mol Sci. 2017;18(4):732-748.

26. Mizushima N, Levine B, Cuervo AM, Klionsky DJ. Autophagy fights disease through cellular self-digestion. Nature. 2008;451(7182): 1069-1075.

27. Ding WX. Role of autophagy in liver physiology and pathophysiology. World J Biol Chem. 2010;1(1):3-12.

28. Sui YQ, Feng YZ. Expression and clinical significance of autophagyrelated genes LC3, Beclin-1 and apoptosis-related genes p53, BCL-2 in colorectal carcinoma. J Clin Exp Pathol. 2012;28:282-286.

29. Mizushima N. Methods for monitoring autophagy. Int J Biochem Cell Biol. 2005;36(12):2491-2502.

30. Kabeya Y, Mizushima N, Ueno T, et al. LC3, a mammalian homologue of yeast Apg8p, is localized in autophagosome membranes after processing. EMBO J. 2000;19(21):5720-5728.

31. Pankiv S, Clausen TH, Lamark T, et al. p62/SQSTM1 binds directly to Atg8/LC3 to facilitate degradation of ubiquitinated protein aggregates by autophagy. J Biol Chem. 2007;282(33):24131-24145.
32. Mai S, Muster B, Bereiterhahn J, Jendrach M. Autophagy proteins LC3B, ATG5 and ATG12 participate in quality control after mitochondrial damage and influence life span. Autophagy. 2012;8(1):47-62.

33. Backer JM. The regulation and function of Class III PI3Ks: Novel roles for Vps34. Biochem J. 2008;410(1):1-17.

34. Klionsky DJ, Abeliovich $\mathrm{H}$, Agostinis $\mathrm{P}$, et al. Guidelines for the use and interpretation of assays for monitoring autophagy in higher eukaryotes. Autophagy. 2015;8(4):445-544.

35. Keren A, Tamir Y, Bengal E. The 38 MAPK signaling pathway: A major regulator of skeletal muscle development. Mol Cell Endocrinol. 2006; 252(1-2):224.

36. Huppertz B, Kingdom JCP. Apoptosis in the trophoblast: Role of apoptosis in placental morphogenesis. J Soc Gynecol Investig. 2004; 11(6):353-362.

37. Davis RJ. Signal transduction by the JNK group of MAP kinases. Cell. 2000;103(2):239.

38. Dhanasekaran DN, Reddy EP. JNK signaling in apoptosis. Oncogene. 2008;27(48):6245-6251.

39. Feng Y, Huang J, Ding Y, Xie F, Shen X. Tamoxifen-induced apoptosis of rat C6 glioma cells via PI3K/Akt, JNK and ERK activation. Oncol Rep. 2010;24(6):1561-1567. 